\title{
Comparison Efficacy of Ultrasound-Guided Needle Release Plus Corticosteroid Injection and Mini-Open Surgery in Patients with Carpal Tunnel Syndrome
}

\section{Zeng Zeng}

Zhejiang Provincial People's Hospital

Nan Lin

Zhejiang Provincial People's Hospital

Cong-Xian Chen ( $\nabla$ kema320@qq.com )

Zhejiang Provincial People's Hospital

\section{Research Article}

Keywords: Ultrasound, Mini-open Surgery, Carpal Tunnel Syndrome, Transverse carpal ligament

Posted Date: November 2nd, 2021

DOI: https://doi.org/10.21203/rs.3.rs-736798/v2

License: () (1) This work is licensed under a Creative Commons Attribution 4.0 International License. Read Full License 


\section{Abstract}

This retrospective study was to compare clinical outcomes of ultrasound-guided needle release with corticosteroid injection versus mini-open surgery in patients with carpal tunnel syndrome (CTS). From January 2020 to December 2020, 40 patients (40 wrists) with CTS were analyzed in this study. Diagnosis was based on clinical symptoms, electro-physiological and ultrasound imaging. 20 wrists were treated with ultrasound-guided needle release plus corticosteroid injection (Group A) and other 20 wrists went mini-open surgery (Group B). We evaluated Boston carpal tunnel questionnaire, electrophysiological parameters (distal motor latency, sensory conduction velocity and sensory nerve action potential of median nerve) and ultrasound parameters (cross-sectional area, flattening ratio and the thicknesses of transverse carpal ligament) both before and 3 months after treatment. Total treatment cost, duration of treatment, healing time and complications were also recorded for two groups. Boston carpal tunnel questionnaire, electrophysiologic and ultrasound outcomes at preoperatively and 3 months postoperatively had significant difference for each group (each $\mathrm{P} \otimes 0.05$ ). There were no complications such as infection, haemorrhage, vascular, nerve or tendon injuries in both groups. Ultrasound-guided needle release and mini-open surgery are both effective measures in treating CTS patients. Ultrasoundguided needle release plus corticosteroid injection provides smaller incision, less cost, less time of treatment and faster recovery compared with mini-open surgery. Ultrasound-guided needle release plus corticosteroid injection is better for clinical application.

\section{Introduction}

Carpal tunnel syndrome (CTS) is the most frequent musculoskeletal disorder of compressive neuropathy [1]. It is caused by compression of the median nerve within the carpal tunnel between carpal bones and transverse carpal ligament (TCL) [2]. CTS usually causes sensory (paresthesia and hypoesthesia), pain, sleep disturbance and motor deficit. It affects about 1-2 men and 4-5 women in every 1,000 persons and always occurs between age 40 to 60 [3]. Mostly, CTS is idiopathic [4]. Sometimes diabetes, pregnancy, amyloidosis and rheumatic synovitis can also cause CTS [4]. In essence, CTS is a multifactorial condition [5]. CTS is diagnose by clinical symptoms and physical examination. Boston carpal tunnel questionnaire, electrophysiological testing and ultrasonography can confirm it as supplementary tools [6-10]. Electrophysiological test of the median nerve is key point in diagnosing CTS [11]. However, when electrophysiological testing is normal in clinically suspected CTS patients, ultrasound imaging seems to be effective in diagnosing [8]. Recently, ultrasound was often used for musculoskeletal diseases, especially in peripheral nerve pathologies and median nerve abnormity $[12,13]$.

There are mainly two types of CTS treatments: surgical and conservative. The choice of treatment depends on the severity of CTS. Conservative treatments are usually for those patients who are in minor and modest circumstances. Conservative treatments include wrist splinting, local corticosteroid injections, laser therapy, therapeutic ultrasound and so on [14]. When conservative ways cannot alleviate CTS symptoms or patients were in severe cases at the beginning, surgical treatment should be preferred [15]. Surgery is also a treatment of choice for persistent or progressive CTS patients, it mainly includes 
dividing TCL in order to relieve median nerve compression [16]. There are 5 kinds of incision size for

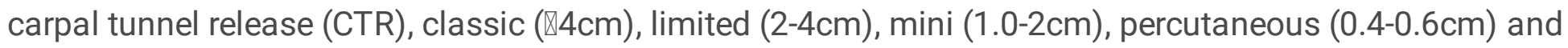
ultra-minimally invasive $(\leq 1 \mathrm{~mm})$ [17-22]. Smaller incision size shows faster recovery time and lower pain $[17,19]$. Although endoscopic CTR has superiority compared with classic open CTR (OCTR), it has damage complications to neurovascular structures and tendons [19]. Cause mini carpal tunnel release has good outcomes in clinical, we use mini incision open surgery when conservative ways cannot alleviate CTS symptoms.

Nowadays, ultrasound-guided corticosteroid injection combined with needle release of the TCL is a new minimally invasive method to treat CTS. Under ultrasound guidance, it is accurately to identify anomalous anatomy thus avoiding damage median nerve surrounding tissues. We can observe the median nerve cross-sectional area reduction to demonstrate effectiveness of corticosteroid injection into the carpal tunnel [23]. The purpose of our study was to evaluate the efficacy of surgery and ultrasoundguided corticosteroid injection combined with needle release.

\section{Methods}

\section{Patients}

Our study was approved by the ethical and scientific review board of Zhejiang Provincial People's Hospital. Written informed consent was obtained from every patient. Between January 2020 and December 2020, we included 40 patients ( 5 men, 35 women) who were diagnosed as CTS in this retrospective study. CTS diagnosis standard was made by clinical history, physical examination, ultrasound and electrophysiological evaluation. Clinical history included tests of sensibility and muscle strength, questioning symptoms of sensory and thenar atrophy examinations. We checked Phalen's test and Tinel's sign on percussion of the wrist. The criteria of CTS in electrophysiological were median distal motor nerve latency $\mathbb{4} 4.2 \mathrm{~ms}$ (stimulation, $2 \mathrm{~cm}$ proximal to the wrist crease) or median sensory nerve conduction velocity $₫ 45 \mathrm{~m} / \mathrm{s}$ (between the $2 \mathrm{~cm}$ proximal to the wrist crease and middle crease of the long finger).

Exclusion criteria were poor general physical condition, pregnancy, carpal fractures, contraindicate for corticosteroid drugs, previous wrist surgery and previous treatment of CTS. 20 patients (Group A) underwent ultrasound-guided corticosteroid injection combined with needle release and other 20 patients (Group B) had mini-open surgery. Follow-up time was 3 months. All patients finished Boston Carpal Tunnel Questionnaire, ultrasound and electrophysiological examination before and 3 months after treatment.

\section{Ultrasound-guided treatment procedures}


Ultrasound-guided treatment as an outpatient procedure was routinely performed in the ultrasound interventional room. Ultrasound examinations were performed by a senior ultrasound doctor using the Mindray Resona7 with a 5-12MHz transducer (Mindray, Shenzhen, China). $5 \mathrm{~mL}$ disposable sterile syringe (with needle) was used for administration of local anesthesia and release TCL.

The patient sit in a chair and affected hand was positioned to the side with the palm up in extended position. All treatment procedures were performed by C.C.X with the probe covered with surgical gloves. Acoustic coupling agent was used on the probe inside of the surgical gloves. Patient's skin was disinfected with complex iodine for 3 times, ranging from metacarpophalangeal joint to $5 \mathrm{~cm}$ above the carpal canal. Place the probe on the wrist with longitudinal section then long axis of median nerve will be clearly shown. The insertion point is $0.5 \mathrm{~cm}$ proximal to the compression point of the median nerve. The direction of the needle entry is from the proximal to distal end of the median nerve. Try to avoid stabbing the median nerve, radial artery and ulnar neurovascular bundle when inserting the needle. Wipe the needle entry point with complex iodine cotton balls, under ultrasound guidance, $4 \mathrm{~mL}$ mixture solution which contains $2 \mathrm{~mL} 2 \%$ lidocaine and $2 \mathrm{~mL} 0.9 \%$ sodium chloride (in a ratio of $1: 1$ ) was injected. Then local anaesthesia layer by layer was performed to the median nerve surface. Under the continuous guidance of ultrasound, acupuncture compression of the TCL at the median nerve continuously, from proximal to distal and from shallow to deep. The operation was completed until there was no resistance to the acupuncture of TCL. Under real-time ultrasound guidance, $2 \mathrm{~mL}$ mixture solution which contained $1 \mathrm{~mL} 2 \%$ lidocaine and $1 \mathrm{~mL}$ betamethasone (Schering Pharmaceutical Co., Ltd. in Shanghai, China) (in a ratio of 1:1) was injected, mixture solution could be seen diffusing in the carpal tunnel (Figure 1). After the needle was pulled out, pressed the needle path by hand for 5 minutes, and then pasted a adhesive bandage at the insertion point. To prevent infection, the wound must keep dry for 48 hours. The whole process lasts about 5-10 minutes. During the operation, all patients had no pain or discomfort. There were no postoperative complications, and the patient was reexamined 3 months after the operation.

\section{Mini-Open surgery procedures}

The patient was placed in supine position under general anesthesia. A pneumatic tourniquet was set at $40 \mathrm{kpa}$ pressure, total time of 1 hour and interval 15 minutes. The size of the incision was about $2 \mathrm{~cm}$ and the shape of it was longitudinal. The skin and subcutaneous tissues were cut open in turn to expose the carpal canal, the thickened and adherent transverse carpal ligament was cut open, the epineurium and bundle membrane of the nerve were loosened, the nerve bundle decompression was performed, and the wound was rinsed after complete hemostasis (Figure 2). After checking nerve release, reclosed layer by layer until to the skin. 


\section{Boston carpal tunnel questionnaire, ultrasonic and electrophysiological evaluations before and after treatment}

Boston Carpal Tunnel Questionnaire is the most used to assess symptom severity and functional status. It includes two parts: Symptom Severity Scale (SSS) and Functional Status Scale (FSS) [6]. SSS has 11 questions and FSS contains 8 items. According to mean score, patients are divided into five grades: minimal ( $0.1-1$ point), mild (1.1-2 points), moderate (2.1-3 points), severe (3.1-4 points) and extreme (4.1-5 points).

In ultrasound examinations, median nerve's flattening ratio (FR) and cross-sectional area (CSA) were measured according to El Miedany and colleagues' grading system [24]. Depending on CSA of median nerve at inlet, severity is divided into follows: mild: $10-13 \mathrm{~mm}^{2}$, moderate: $>13-15 \mathrm{~mm}^{2}$, Severe: $>15 \mathrm{~mm}^{2}$ [25]. FR was the ratio of the nerve's transverse axis to the anteroposterior axis. It was assessed at the level of the pisiform bone. Transverse carpal ligament (TCL) was measured on the cross-section at the level of hamate bone.

In electrophysiologic examinations, we recorded the median nerve's distal motor latency (DML), sensory conduction velocity (SCV) and sensory nerve action potential (SNAP). According to recommendations of the American Association of Neuromuscular and Electrodiagnostic Medicine [26] and American Academy of Orthopedic Surgeon work group (AAOS) [27], CTS severity was classified as follows: negative: normal findings of all tests (both comparative and segmental studies), minimal: abnormal on comparative or segmental tests, mild: normal DML with finger-wrist tract SNCV slowed, moderate: increased DML with finger-wrist tract SNCV slowed, severe: increased DML with finger-wrist tract absence of sensory response, extreme: thenar motor absence of response.

\section{Statistical analysis}

Statistical analyses were performed using SPSS version 22.0 (SPSS Inc., Chicago, IL, USA). All quantitative data were expressed as mean \pm standard deviations (SD). All qualitative data were expressed as number and percentage. We used student $t$ test to compare quantitative data between groups and Mann-Whitney $U$ test to compare qualitative data. $P$ values $₫ 0.05$ were considered statistically significant.

\section{Results}

In our study, we included 40 eligible patients ( 40 wrists) with CTS. There were no significant differences

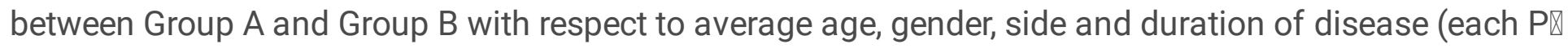
0.05) (Table 1). For the treatment duration, total treatment cost and healing time, Group A had 
significance compare with Group B (each Pख0.05). Group A had shorter treatment duration, cost less and had quicker healing time compared with Group B. There were no complications such as infection, haemorrhage, vascular, nerve or tendon injuries in both groups. Patients had no recurrent symptoms after treatment in 3 months.

Table 2 was Boston Carpal Tunnel Questionnaire before and 3 months after treatment for two groups. It showed significant difference for both two groups with respect to symptom severity scale (SSS) and functional status scale (FSS) before and 3 months after treatment (each Pख0.05). There was no significance regarding SSS and FSS between two groups 3 months after treatment $(P=0.73)$.

Results of electrophysiologic examinations for two groups before and 3 months after treatment were shown in Table 3. There was no significant difference with respect to DML, SCV and SNAP before treatment between two groups (each $\mathrm{P} \otimes 0.05$ ). Both two groups showed significance in their DML, SCV and SNAP 3 months after treatment which suggested that both ultrasound-guided needle release plus corticosteroid injection and mini-open surgery had the effectiveness in treating CTS (each Pख0.05). Furthermore, in terms of DML and SCV 3 months after treatment, there was significant difference for two groups (each $\mathrm{P} \otimes 0.05$ ). It suggested that ultrasound-guided needle release plus corticosteroid injection was superior to mini-open surgery in the matter of DML and SCV.

Ultrasound examinations results of both groups before and after treatment were shown in Table 4. There was no significant difference with respect to FR, CSA and TCL before treatment between two groups (each $\mathrm{P} \otimes 0.05$ ). There was significant difference in terms of the before and 3 months after treatment in the values of FR, CSA and TCL for both groups (each $P \otimes 0.05$ ), which indicated that the effectiveness of two therapeutic approaches. In addition, we found that there was no significant difference between two groups in the values of FR, CSA and TCL 3 months after treatment.

\section{Discussion}

CTS is usually diagnosed by illness history and physical examination [28]. Electrophysiological examination is often used to confirm it [29]. Ultrasound as a new way to diagnose CTS has now be widely used in clinic because it is easy and noninvasive [30]. The principle of treating CTS is the decompression of carpal tunnel by opening the median nerve's flattening ratio. This is usually done by using conventional open surgery (incision size about $5 \mathrm{~cm}$ ), mini-open surgery (incision size about $2 \mathrm{~cm}$ ) or endoscopy (incision size about $1.5 \mathrm{~cm}$ ) [11]. Jugovac et al. found that compared with conventional open surgery, mini-open surgery had smaller incision and return to work in shorter time (i.e. 15 days versus 30 days) [31]. In 1990s, endoscopy came into effect. It is a safe technique that reduces morbidity, postoperative 
pain and recovery time [11]. However, endoscopy has its own shortcomings, potential risk of injury to adjacent neurovascular structures [32]. Nowadays, a new procedure which uses a needle to release the nerve based on ultrasound as guidance came into use in clinic. Compared with open surgery and endoscopic treatments, ultrasound guided needle release allows for a fast recovery and an easy postoperative care due to almost no wound.

Our research found CTS was more common in women cause we retrospectively studied 40 CTS patients, 35 of them were female. That was consistent with study of Tang et al. and Baysal et al. where they found that hand-intensive housewives had higher incidence of CTS $[33,34]$. The higher incidence of CTS in women may due to physiologic difference, hormonal changes and intense housework [35]. The mean age of Group A and B patients were $54.05 \pm 9.40$ and $53.70 \pm 8.22$ respectively. CTS incidence peaks for individuals were those people ages of 40 to 60 [3].

In our study, we compared the clinical outcomes of ultrasound-guided needle release plus corticosteroid injection and mini-open surgery and found that both two groups had significant improvement with respect to $B C T Q$, electrophysiological and ultrasound results after treatment. Both two groups had no recurrent symptoms or pain syndrome 3 months after treatment. These findings indicated that ultrasound-guided needle release plus corticosteroid injection and mini-open surgery had the same effect in treatment. However, in terms of cost, operation time, recovery time and recurrence, ultrasound-guided needle release plus corticosteroid injection is better than mini-open surgery. Furthermore, ultrasoundguided needle release plus corticosteroid injection has almost no scar and was deemed to be more preferable in terms of cosmesis.

In Group A patients, no complications were reported during or after treatment due to proper visualization of carpal tunnel structure under ultrasound guidance. Recent studies demonstrated that corticosteroid injection under ultrasound guidance is better than blind injection $[17,31,36]$. The advantage of ultrasound guidance can be clearly listed as follows: 1) the structure can be seen so that doctor know the location and reach to the carpal tunnel and inject medicine without damaging neighbor tissue; 2) great degree of needle control: the doctor can change the distance of the needle and median nerve in visualization so that the needle can reach close to median nerve; 3 ) ensure uniformly distribute injection solution around the carpal tunnel and peel median nerve away from adhesions [37, 38]. Studies had shown that the injection of corticosteroids was widely used in the field of pain management because it can reduce pain and swelling. In CTS patients, after injecting corticosteroids into flexor pollicis longus tendon sheath and common flexor sheath can relieve pain and improve functional impairment [39-40].

During ultrasound-guided needle release plus corticosteroid injection, the following points should be noted: 1) The puncture was carried out along the long axis of the median nerve. During the process of releasing the transverse carpal ligament by acupuncture, attention should be paid to protect the median nerve deep in the transverse carpal ligament. 2) Due to the puncture space of carpal tunnel is relatively small, appropriate wrist extension can increase the puncture space. The puncture angle should not be too large in the process of puncture, $15^{\circ}-30^{\circ}$ with the skin of wrist is suitable. 3) Due to the density of the 
transverse carpal ligament, when the lesion occurs, it will be more tenacious. There will be a sense of resistance during acupuncture, and when the sense of resistance disappears, it indicates that the release is thorough. 4) The puncture should be carried out in the plane so that the needle tip can be observed at all times to avoid injury to the median nerve. Finally, the mixture of lidocaine and betamethasone should not be injected into the transverse carpal ligament during the injection into the carpal canal. When the relationship between the tip and median nerve is not clear, a small amount of normal saline can be injected.

There are several limitations of our study. First of all, the sample size is small, more data are needed for further study. Secondly, we only compared ultrasound-guided needle release plus corticosteroid injection with mini-open surgery, it is still not clear how effective it compared with endoscopic or other treatment procedures. Furthermore, we only select the 3 months follow-up because some patients return to work after treatment, many factors may affect the outcome of follow-up. The long-term effects are still worth watching.

\section{Conclusion}

Both ultrasound-guided needle release plus corticosteroid injection and mini-open surgery had great benefit in the treatment of CTS. Ultrasound-guided needle release plus corticosteroid injection had advantages of shorter operation time, quicker recovery time, smaller wound and less cost compared with mini-open surgery. According to those advantages, ultrasound-guided needle release plus corticosteroid injection is better for clinical application.

\section{References}

1. Alfonso, C. et al. Diagnosis, treatment and follow-up of the carpal tunnel syndrome: a review. Neurol Sci, 31, 243-252 (2010).

2. Katz, J. N. \& Simmons, B. P. Clinical practice. Carpal tunnel syndrome. N Eng/ J Med, 346, 1807-1812 (2002).

3. Ghasemi-Rad, M. et al. A handy review of carpal tunnel syndrome: From anatomy to diagnosis and treatment. World J Radiol, 6, 284-300 (2014).

4. Yoshii, Y., Zhao, C. \& Amadio, P. C. Recent Advances in Ultrasound Diagnosis of Carpal Tunnel Syndrome. Diagnostics (Basel), 10, 596 (2020).

5. Genova, A. et al. Carpal Tunnel Syndrome: A Review of Literature. Cureus, 12, e7333 (2020).

6. Levine, D. W. et al. Self-administered questionnaire for the assessment of severity of symptoms and functional status in carpal tunnel syndrome. J Bone Joint Surg Am, 75, 1585-1592 (1993).

7. Padua, L. et al. Carpal tunnel syndrome: ultrasound, neurophysiology, clinical and patient-oriented assessment. Clin Neurophysiol, 119, 2064-2069 (2008).

8. Rahmani, M. et al. The ultrasonographic correlates of carpal tunnel syndrome in patients with normal electrodiagnostic tests. Radiol Med, 116, 489-496 (2011). 
9. Granata, G. et al. Prevalence of bifid median nerve at wrist assessed through ultrasound. Neurol Sci, 32, 615-618 (2011).

10. Chen, Y. T. et al. Review of ultrasonography in the diagnosis of carpal tunnel syndrome and a proposed scanning protocol. J Ultrasound Med, 35, 2311-2324 (2016).

11. Petrover, D. \& Richette, P. Treatment of carpal tunnel syndrome: from ultrasonography to ultrasound guided carpal tunnel release. Joint Bone Spine, 85, 545-552 (2018).

12. Roomizadeh, P. et al. Ultrasonographic Assessment of Carpal Tunnel Syndrome Severity: A Systematic Review and Meta-Analysis. Am J Phys Med Rehabil, 98, 373-381 (2019).

13. Karahan, A. Y. et al. Superb Microvascular Imaging of the Median Nerve in Carpal Tunnel Syndrome: An Electrodiagnostic and Ultrasonographic Study. J Ultrasound Med, 37, 2855-2861 (2018).

14. Wipperman, J. \& Goerl, K. Carpal Tunnel Syndrome: Diagnosis and Management. Am Fam Physician, 94, 993-999 (2016).

15. Burton, C., Chesterton, L. S. \& Davenport, G. Diagnosing and managing carpal tunnel syndrome in primary care. Br J Gen Pract, 64, 262-263 (2014).

16. Crnković, T. et al. Carpal tunnel and median nerve volume changes after tunnel release in patients with the carpal tunnel syndrome: a magnetic resonance imaging (MRI) study. Int Orthop, 40, 981987 (2016).

17. Nakamichi, K. et al. Percutaneous carpal tunnel release compared with mini-open release using ultrasonographic guidance for both techniques. J Hand Surg Am, 35, 437-445 (2010).

18. Klein, R. D., Kotsis, S. V. \& Chung, K. C. Open carpal tunnel release using a 1-centimeter incision: technique and outcomes for 104 patients. Plast Reconstr Surg, 111, 1616-1622 (2003).

19. Nakamichi, K. \& Tachibana, S. Ultrasonographically assisted carpal tunnel release. J Hand Surg Am, 22, 853-862 (1997).

20. Lecoq, B. et al. Ultrasound-guided percutaneous surgery for carpal tunnel syndrome: a cadaver study. Joint Bone Spine, 78, 516-518 (2011).

21. Rowe, N. M. et al. Sonographically guided percutaneous carpal tunnel release: an anatomic and cadaveric study. Ann Plast Surg, 55, 52-56 (2005).

22. Rojo-Manaute, J. M. et al. Ultra-minimally invasive sonographically guided carpal tunnel release: anatomic study of a new technique. J Ultrasound Med, 32, 131-142 (2013).

23. Cartwright, M. S. et al. Median nerve changes following steroid injection for carpal tunnel syndrome. Muscle Nerve, 44, 25-29 (2011).

24. El Miedany, Y. M., Aty, S. A. \& Ashour, S. Ultrasonography versus nerve conduction study in patients with carpal tunnel syndrome: substantive or complementary tests?, 43, 887-895 (2004).

25. Giannini, F. et al. A new clinical scale of carpal tunnel syndrome: validation of the measurement and clinical-neurophysiological assessment. Clin Neurophysiol, 113, 71-77 (2002).

26. England, J. D. et al. American Academy of Neurology; American Association of Neuromuscular and Electrodiagnostic Medicine; American Academy of Physical Medicine and Rehabilitation. Practice 
parameter: the evaluation of distal symmetric polyneuropathy: the role of laboratory and genetic testing (an evidence-based review). Report of the American Academy of Neurology, the American Association of Neuromuscular and Electrodiagnostic Medicine, and the American Academy of Physical Medicine and Rehabilitation. PM R, 1, 5-13 (2009).

27. Keith, M. W. et al. Diagnosis of carpal tunnel syndrome. J Am Acad Orthop Surg, 17, 389-396 (2009).

28. Jablecki, C. K. et al. American Association of Electrodiagnostic Medicine; American Academy of Neurology; American Academy of Physical Medicine and Rehabilitation. Practice parameter: Electrodiagnostic studies in carpal tunnel syndrome. Report of the American Association of Electrodiagnostic Medicine, American Academy of Neurology, and the American Academy of Physical Medicine and Rehabilitation. Neurology, 58, 1589-1592 (2002).

29. Kanikannan, M. A. et al. Comparison of high-resolution sonography and electrophysiology in the diagnosis of carpal tunnel syndrome. Ann Indian Acad Neurol, 18, 219-225 (2015).

30. Alemán, L. et al. Reproducibility of sonographic measurements of the median nerve. $J$ Ultrasound Med, 27, 193-197 (2008).

31. Jugovac, I. et al. Carpal tunnel release by limited palmar incision vs traditional open technique: randomized controlled trial. Croat Med J, 43, 33-36 (2002).

32. Sayegh, E. T. \& Strauch, R. J. Open versus endoscopic carpal tunnel release: a meta-analysis of randomized controlled trials. Clin Orthop Relat Res, 473, 1120-1132 (2015).

33. Tang, X., Zhuang, L. \& Lu, Z. Carpal tunnel syndrome: a retrospective analysis of 262 cases and a one to one matched case-control study of 61 women pairs in relationship between manual housework and carpal tunnel syndrome. Chin Med J (Engl), 112, 44-48 (1999).

34. Baysal, O. et al. Comparison of three conservative treatment protocols in carpal tunnel syndrome. Int J Clin Pract, 60, 820-828 (2006).

35. Wilgis, E. F. Treatment options for carpal tunnel syndrome. JAMA, 288, 1281-1282 (2002).

36. Smith, J. et al. Sonographic Visualization of Thenar Motor Branch of the Median Nerve: A Cadaveric Validation Study. PM R, 9, 159-169 (2017).

37. Lee, J. C. \& Healy, J. C. Normal sonographic anatomy of the wrist and hand., 25, 1577-1590 (2005).

38. Ustün, N. et al. Ultrasound-guided vs. blind steroid injections in carpal tunnel syndrome: A single-blind randomized prospective study. Am J Phys Med Rehabil, 92, 999-1004 (2013).

39. Ly-Pen, D. et al. Comparison of surgical decompression and local steroid injection in the treatment of carpal tunnel syndrome: 2-year clinical results from a randomized trial., 51, 1447-1454 (2012).

40. Huisstede, B. M. et al. Carpal tunnel syndrome: hand surgeons, hand therapists, and physical medicine and rehabilitation physicians agree on a multidisciplinary treatment guideline-results from the European HANDGUIDE Study. Arch Phys Med Rehabil, 95, 2253-2263 (2014).

\section{Declarations}

\section{Author contributions}


Guarantor of integrity of entire study, Z.Z. and C.X.C.; study concepts/ study design or data acquisition or data analysis/ interpretation, Z.Z., C.X.C. and N.L.; manuscript drafting or manuscript revision for important intellectual content, Z.Z. and C.X.C.; approval of final version of submitted manuscript, Z.Z., C.X.C. and N.L.; agrees to ensure any questions related to the work are appropriately resolved, Z.Z., C.X.C. and N.L..

\section{Additional Information}

The authors had no conflict of interests to declare in relation to this paper.

\section{Tables}

Table 1 Clinical data of two groups

\begin{tabular}{|c|c|c|c|c|}
\hline & & $\begin{array}{l}\text { Group A } \\
\text { (n=20 wrists) }\end{array}$ & $\begin{array}{l}\text { GroupB } \\
\text { (n=20 wrists) }\end{array}$ & P-value \\
\hline Number of patients & & 20 & 20 & NA \\
\hline \multirow[t]{3}{*}{ Gender } & & & & 0.633 \\
\hline & Male & 3 & 2 & \\
\hline & Female & 17 & 18 & \\
\hline \multirow[t]{3}{*}{ Side } & & & & 0.507 \\
\hline & Right & 12 & 14 & \\
\hline & Left & 8 & 6 & \\
\hline Age (years) & & $54.05 \pm 9.40$ & $53.70 \pm 8.22$ & 0.927 \\
\hline Duration of CTS (months) & & $19.05 \pm 15.74$ & $10.50 \pm 7.98$ & 0.526 \\
\hline Duration of treatment (minutes) & & $5.75 \pm 1.37$ & $19.00 \pm 1.30$ & $0.000^{*}$ \\
\hline Total treatment cost (dollars) & & $54.30 \pm 3.38$ & $644.55 \pm 58.49$ & $0.021^{*}$ \\
\hline Healing time (months) & & $1.16 \pm 0.14$ & $2.44 \pm 0.24$ & $0.000^{*}$ \\
\hline
\end{tabular}

*, P-value 0.05

Table 2 Boston Carpal Tunnel Questionnaire before and 3 months after treatment for two groups 


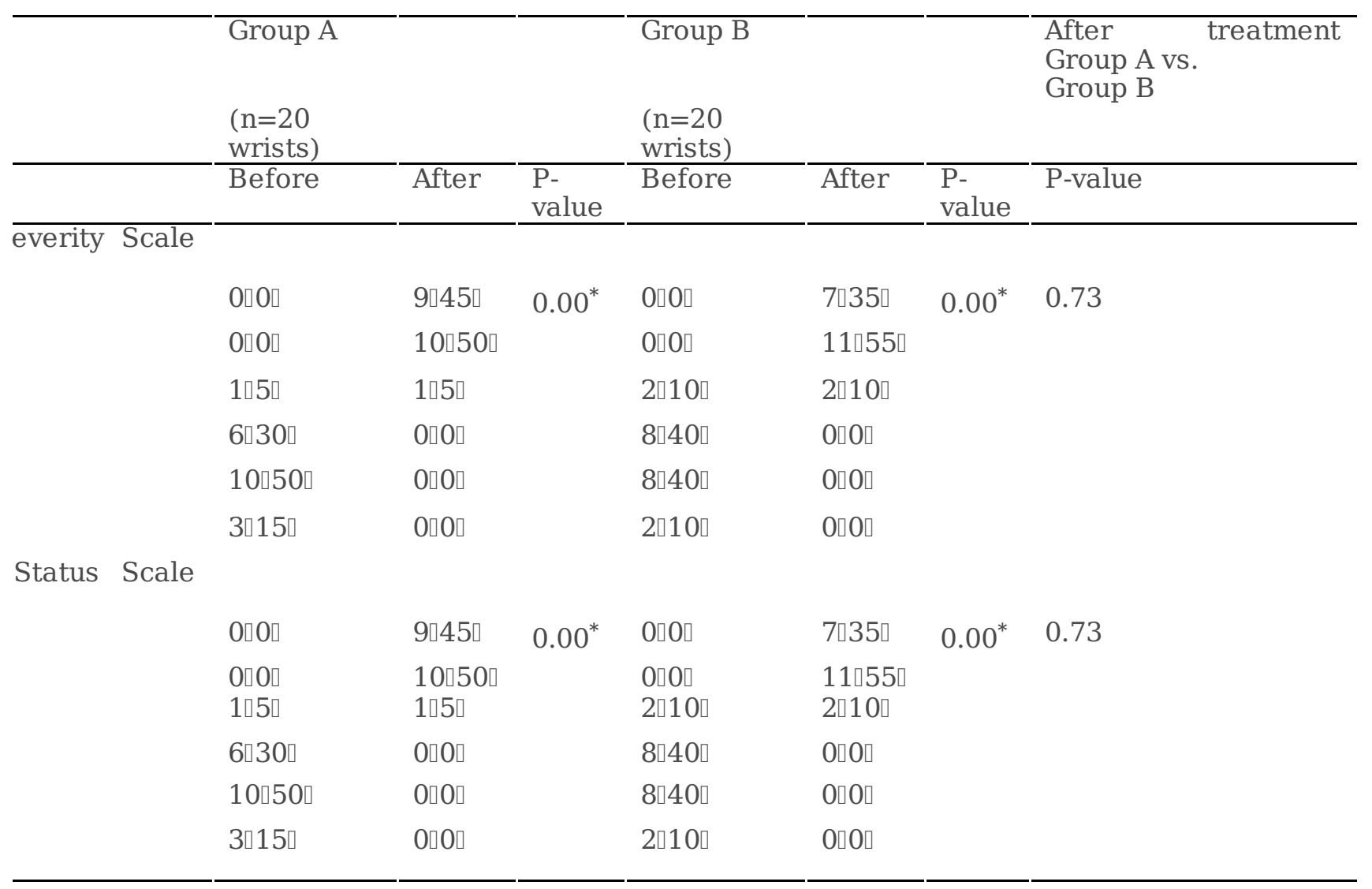

*, P-value 0.05

Table 3 Electrophysiologic results before and 3 months after treatment for two groups

\begin{tabular}{|c|c|c|c|}
\hline \multicolumn{2}{|l|}{$\begin{array}{l}\text { Group A } \\
\text { ( } \mathrm{n}=20 \text { wrists) }\end{array}$} & \multicolumn{2}{|l|}{$\begin{array}{l}\text { Group B } \\
\text { (n=20 wrists) }\end{array}$} \\
\hline Before & After & Before & After \\
\hline $4.81 \pm 1.85$ & $3.50 \pm 1.83^{+\#}$ & $4.80 \pm 0.92$ & $3.80 \pm 0.89^{+}$ \\
\hline $40.65 \pm 5.75$ & $51.50 \pm 4.51^{+\#}$ & $38.69 \pm 3.09$ & $50.11 \pm 6.21^{+}$ \\
\hline $7.24 \pm 3.30$ & $17.42 \pm 7.31^{+}$ & $7.10 \pm 5.75$ & $19.53 \pm 6.76^{+}$ \\
\hline
\end{tabular}

+ vs. Before treatment, P-value匹0.05.

\# vs. Group B after treatment, P-value₫0.05.

Table 4 Ultrasound results before and 3 months after treatment for two groups 


\begin{tabular}{|c|c|c|c|c|}
\hline & $\begin{array}{l}\text { Group A } \\
\text { (n=20 } \\
\text { wrists) }\end{array}$ & & $\begin{array}{l}\text { Group B } \\
\text { (n=20 } \\
\text { wrists) }\end{array}$ & \\
\hline & Before & After & Before & After \\
\hline ) at the level of hamate & $2.89 \pm 0.36$ & $2.31 \pm 0.20^{\S}$ & $2.88 \pm 0.39$ & $2.34 \pm 0.18^{\S}$ \\
\hline $\mathrm{l}$ area at the level of pisiform bone & $0.15 \pm 0.02$ & $0.09 \pm 0.01^{\S}$ & $0.14 \pm 0.03$ & $0.09 \pm 0.02^{\S}$ \\
\hline $\begin{array}{l}\text { trpal ligament-Thickness on the cross- } \\
\text { evel of hamate bone (TCL, } \mathrm{mm} \text { ) }\end{array}$ & $4.30 \pm 0.34$ & $3.45 \pm 0.30^{\S}$ & $4.10 \pm 0.43$ & $3.48 \pm 0.41^{\S}$ \\
\hline
\end{tabular}

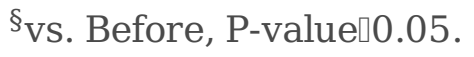

\section{Figures}




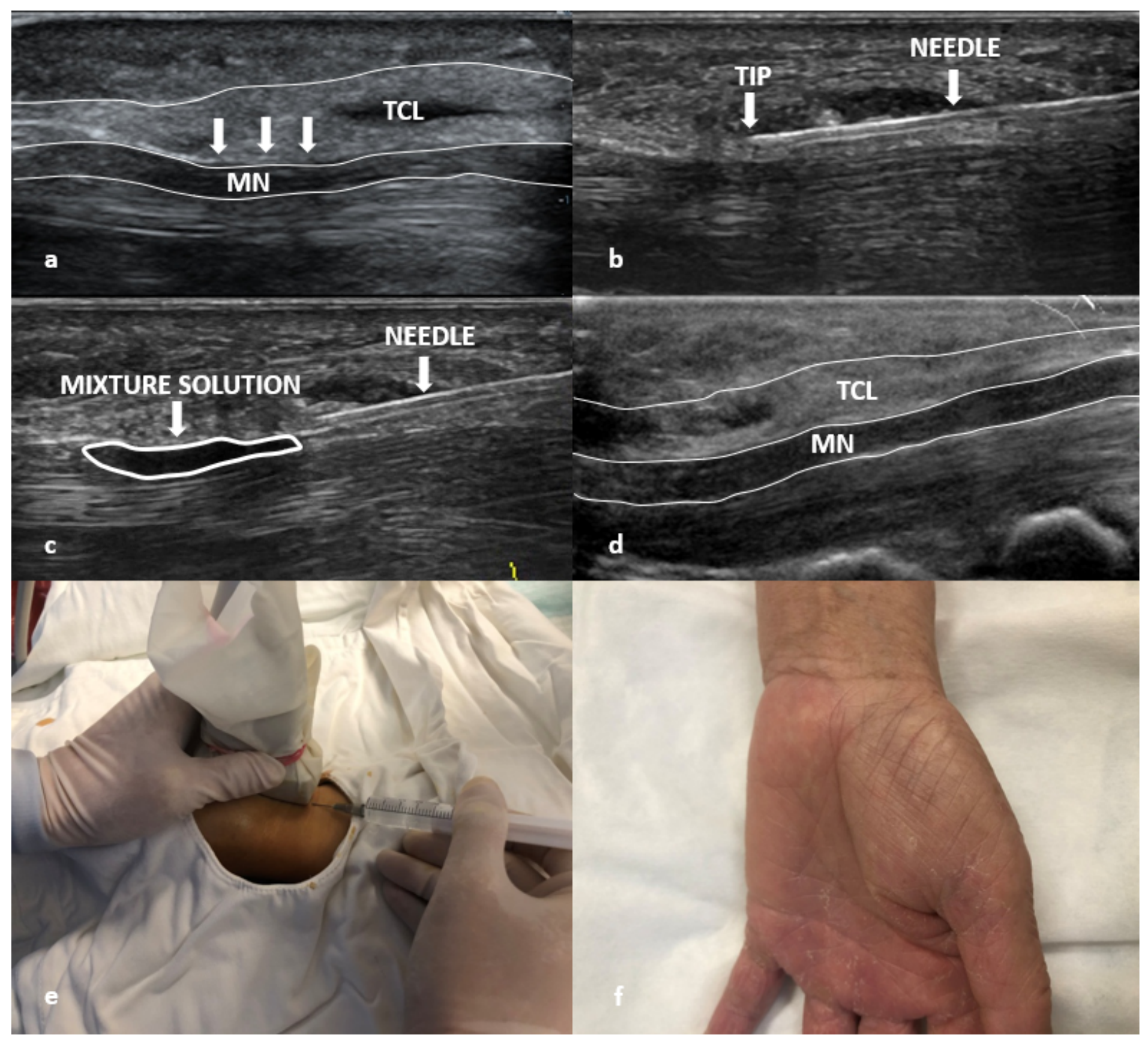

\section{Figure 1}

Ultrasound-guided needle release plus corticosteroid injection procedure of a Group A patient. a) The transverse carpal ligament (TCL) was obviously compressed (white arrow) in ultrasound image before treatment. b) acupuncture process c) $2 \mathrm{~mL}$ mixture solution (white ellipse) which contained $1 \mathrm{~mL} 2 \%$ lidocaine and $1 \mathrm{~mL}$ betamethasone was injected into the carpal tunnel. d) TCL was normal (no compression) after 3 months follow-up in ultrasound image. e) Sonographer held the probe covered with surgical gloves with one hand and acupuncture compression of the TCL at the median nerve continuously under continuous guidance of ultrasound. f) Postoperative image (no scar). 

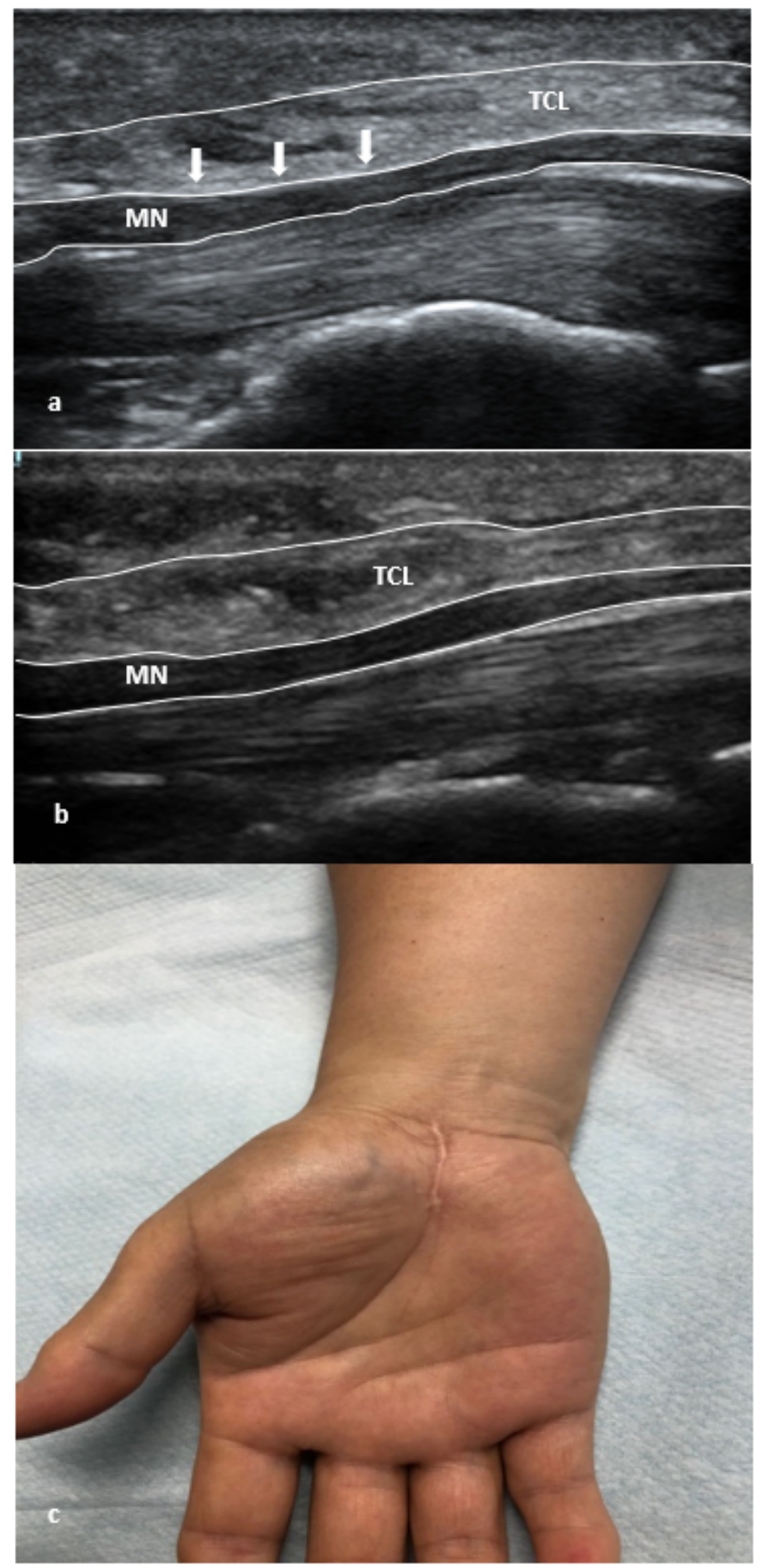

\section{Figure 2}

Mini-Open surgery of a Group B patient. a) The transverse carpal ligament (TCL) was obviously compressed (white arrow) in ultrasound image before treatment. b) TCL was normal (no compression) after 3 months follow-up in ultrasound image. c) Postoperative image (with a $2 \mathrm{~cm}$ scar). 